\title{
Collider signals of gravitino dark matter in bilinearly broken R-parity
}

\section{Martin Hirsch*}

Instituto de Fisica Corpuscular-CSIC, Valencia, Spain

E-mail: mahirsch@ific.uv.es

In supersymmetric models with violation of R-parity the lightest supersymmetric particle decays. Thus, the usual SUSY candidate for the dark matter in the universe - the lightest neutralino, $\tilde{\chi}_{1}^{0}$ - is lost. If instead the gravitino is the LSP, as happens, for example, in models with gauge mediated supersymmetry breaking, the gravitino decays with a half-live far exceeding the age of the universe and thus can potentially solve the dark matter problem. Here, the decays of the next-to-lightest supersymmetric particle, assumed to be the neutralino, are considered in such a scenario. In models of bilinear R-parity breaking, data from neutrino oscillation experiments fixes the branching ratio of the decay $\tilde{\chi}_{1}^{0} \rightarrow \tilde{G} \gamma$ in the range $10^{-3}-10^{-2}$, if the gravitino mass is of the order (few) $100 \mathrm{eV}$. The combined hypothesis of bilinear R-parity violation plus (light) gravitino dark matter is therefore directly testable at the next generation of colliders.

International Europhysics Conference on High Energy Physics

July 21st - 27th 2005

Lisboa, Portugal

${ }^{*}$ Talk based on [1]. 


\section{Introduction}

Supersymmetric models with bilinear R-parity violation $\left(\not R_{p}\right)$ can explain [2] current data on neutrino masses and mixings [3] without invoking any GUT-scale physics. However, in $\not \not_{p}$ SUSY the lightest supersymmetric particle decays. For all superpartners of standard model particles these decays proceed at rates that even the most tiny amount of $\not \not_{p}$ rules out MSSM particles as dark matter. A light gravitino, on the other hand, couples so weakly to standard model particles that its half-live far exceeds the age of the universe even for $\not \mathbb{R}_{p}$ couplings as large as $\mathscr{O}(1)$ [4]. Thus, contrary to popular believe, supersymmetry holds the promise to solve the dark matter problem even if R-parity is violated.

Gravitinos are expected to be the LSP in models of gauge mediated supersymmetry breaking (GMSB) [5], with masses of the order of $m_{3 / 2} \simeq \frac{1}{k} \sqrt{F} /(1000 \mathrm{TeV})^{2} 0.24 \mathrm{keV}$, where $k$ is a model dependent factor and $F$ is the scale of supersymmetry breaking.

If gravitinos were in thermal equilibrium in the early universe, their contribution to the matter content of the universe can be estimated to be [6]

$$
\Omega_{3 / 2} h^{2} \simeq 0.11 \mathscr{F}\left(\frac{m_{3 / 2}}{100 \mathrm{eV}}\right)\left(\frac{100}{g_{*}}\right) .
$$

Here, $\Omega_{3 / 2}$ is the density of gravitinos in units of the critical density, $h$ is the Hubble parameter in units of $100 \mathrm{~km} \mathrm{~s}^{-1} \mathrm{Mpc}^{-1}$ and $g_{*}$ is the effective number of degrees of freedom at the time of gravitino decoupling. Current data give the dark matter density of the universe [7] as $\Omega_{D M} h^{2} \simeq$ $0.111 \pm 0.006 . \mathscr{F}$ in eq. (1.1) is a factor smaller than 1 indicating possible departures from the standard thermal history of the universe due to additional entropy production after the time of gravitino decoupling $[8,9]$.

In the rest of this short talk, the collider phenomenology of such a light gravitino within $\not_{p}$ models will be briefly discussed.

\section{Preliminaries}

In $\not R_{p}$ SUSY the neutralino will decay to three SM fermion final states or, if kinematically allowed, into gauge bosons and leptons, $W^{ \pm} l^{\mp}$ and $Z^{0} v$. Consider first the decay to gauge bosons. In GMSB scenarios the lightest neutralino is usually bino dominated [5]. Binos couple to gauge bosons proportional to $\Lambda_{i}=\varepsilon_{i} \mathrm{v}_{d}+\mathrm{v}_{i} \mu \sim \sqrt{m_{v}^{\text {Tree }}}$. Using expressions from [10] it can be estimated

$$
\Gamma\left(\tilde{\chi}_{1}^{0} \rightarrow \sum_{i} W^{ \pm} l^{\mp}\right) \sim \frac{g^{2} g^{2} M_{2} m_{\tilde{\chi}_{1}^{0}}}{\left(16 \pi M_{1} m_{\gamma}\right)} f\left(m_{W}^{2} / m_{\tilde{\chi}_{1}^{0}}^{2}\right) m_{v}^{\text {Tree }} .
$$

Here, $f(x)$ is a phase space factor, see [1]. A similar expression holds for $\Gamma\left(\tilde{\chi}_{1}^{0} \rightarrow \sum_{i} Z^{0} v_{i}\right)$ with an additional prefactor of $1 /\left(4 c_{W}^{2}\right)$. Assuming $M_{1} \simeq M_{2} / 2$ and $|\mu| / M_{1} \simeq 4$, as is typical for GMSB models, results very roughly in $\Gamma \simeq 2 \times 10^{-4}\left(\frac{m_{v}}{0.05 \mathrm{eV}}\right) f\left(m_{W}^{2} / m_{\tilde{\chi}_{1}^{0}}^{2}\right) \mathrm{eV}$.

Neutralino decays to three fermions can also be mediated by scalar quark and scalar lepton exchange. With approximate scalar lepton couplings from [11] we estimate that the decay $\Gamma\left(\tilde{\chi}_{1}^{0} \rightarrow\right.$ $\left.v \tau^{ \pm} l^{\mp}\right)$ is very roughly of order [1]

$$
\Gamma\left(\tilde{\chi}_{1}^{0} \rightarrow v \tau^{ \pm} l^{\mp}\right) \sim \frac{g^{\prime 2} h_{\tau}^{2}}{512 \pi^{3}}\left(\frac{\vec{\varepsilon}}{\mu}\right)^{2}\left(\frac{m_{\tilde{\chi}_{1}^{0}}}{m_{\tilde{\tau}}}\right)^{4} g\left(\frac{m_{\tilde{\chi}_{1}^{0}}^{2}}{m_{\tilde{\tau}}^{2}}\right) m_{\tilde{\chi}_{1}^{0}} .
$$



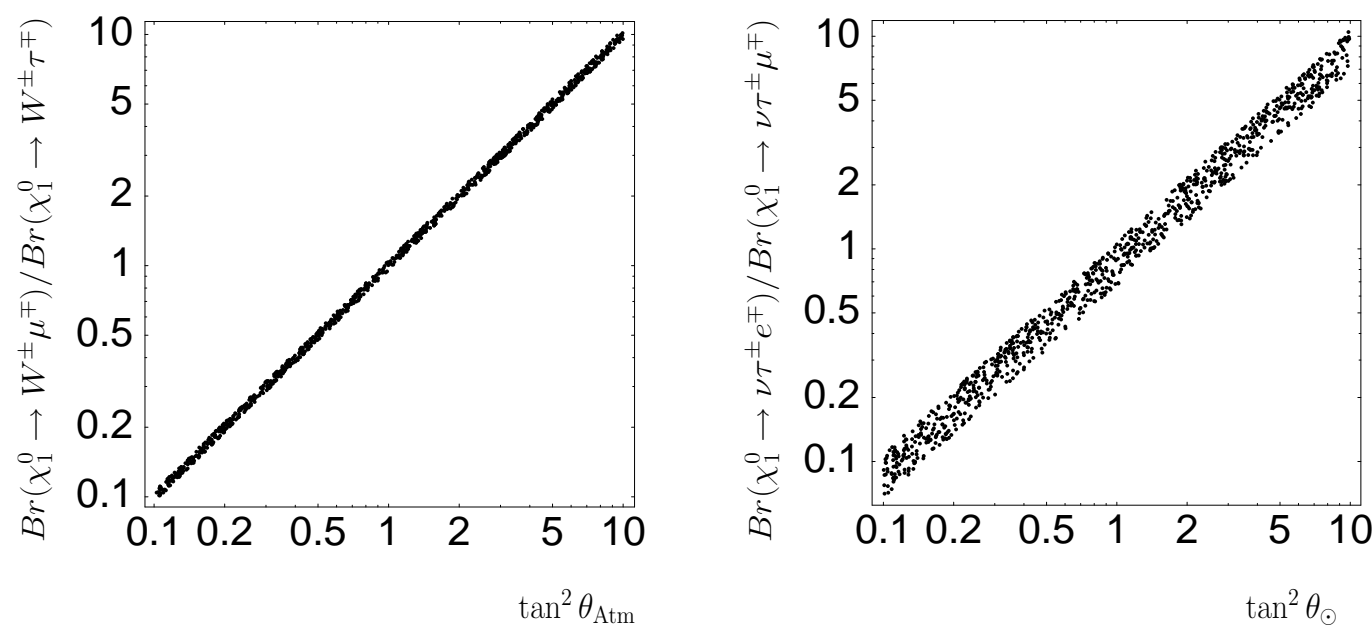

Figure 1: To the left: Ratio of $B R\left(\tilde{\chi}_{1}^{0} \rightarrow W \mu\right) / B R\left(\tilde{\chi}_{1}^{0} \rightarrow W \tau\right)$ as a function of $\tan ^{2} \theta_{A t m}$. To the right: $B R\left(\tilde{\chi}_{1}^{0} \rightarrow \sum_{i} v_{i} \tau e\right) / B R\left(\tilde{\chi}_{1}^{0} \rightarrow \sum_{i} v_{i} \tau \mu\right)$ as function of $\tan ^{2} \theta_{\odot}$. The measured neutrino angles lead to predictions for these observables at future colliders, see text.

Note, that $\Gamma\left(\tilde{\chi}_{1}^{0} \rightarrow v \tau^{ \pm} l^{\mp}\right)$ goes to zero proportional to the fourth power of $m_{\tilde{\chi}_{1}^{0}} / m_{\tilde{\tau}}$. Since in GMSB the (right) scalar tau is never very much heavier than the neutralino, contrary to squarks and other scalar leptons, Eq.(2.2) usually dominates over other Feynman graphs with scalar exchange.

Eq.(2.2) can take a wide range of values. Just to give a flavour of its typical size, for $\frac{|\vec{\varepsilon}|}{\mu} \sim$ $3 \times 10^{-4}, \tan \beta=10$, and $m_{\tilde{\chi}_{1}^{0}}=100 \mathrm{GeV}$ and $m_{\tilde{\tau}}=120 \mathrm{GeV}$ one finds $\Gamma\left(\tilde{\chi}_{1}^{0} \rightarrow v \tau^{ \pm} l^{\mp}\right) \sim 3 \times 10^{-3}$ $\mathrm{eV}$.

The decay width of a neutralino NLSP to gravitino-photon is given by [5]

$$
\Gamma\left(\tilde{\chi}_{1}^{0} \rightarrow \tilde{G} \gamma\right) \simeq 1.2 \times 10^{-6} \kappa_{\gamma}^{2}\left(\frac{m_{\tilde{\chi}_{1}^{0}}}{100 \mathrm{GeV}}\right)^{5}\left(\frac{100 \mathrm{eV}}{m_{3 / 2}}\right)^{2} \mathrm{eV}
$$

Here, $\kappa_{\gamma}=\left|\cos \theta_{W} N_{11}+\sin \theta_{W} N_{12}\right|$. From eqs (2.1), (2.2) and (2.3) we can very roughly estimate a branching ratio of $\operatorname{Br}\left(\tilde{\chi}_{1}^{0} \rightarrow \tilde{G} \gamma\right) \sim 10^{-(2-3)}$ for $m_{3 / 2}$ of $\mathscr{O}(100) \mathrm{eV}$. This is the main result of the current paper.

\section{Numerical results}

Supersymmetric particle spectra have been calculated using the program package SPheno [12]. Neutrino masses (and angles) are calculated following [2]. Only points which fit data from neutrino oscillation experiments [3] and fulfill limits from the negative searches for SUSY particles [7] and the Higgs at LEP [13] are accepted for further analysis. R-parity violating neutralino decays are then treated as described in [10].

In GMSB, typically the most important decay channels of the lightest neutralino are $\tilde{\chi}_{1}^{0} \rightarrow W l_{i}$, $\tilde{\chi}_{1}^{0} \rightarrow Z^{0} v_{i}$ and $\tilde{\chi}_{1}^{0} \rightarrow \sum_{i} v_{i} \tau l_{i}$. These decays make an important consistency check of the model 

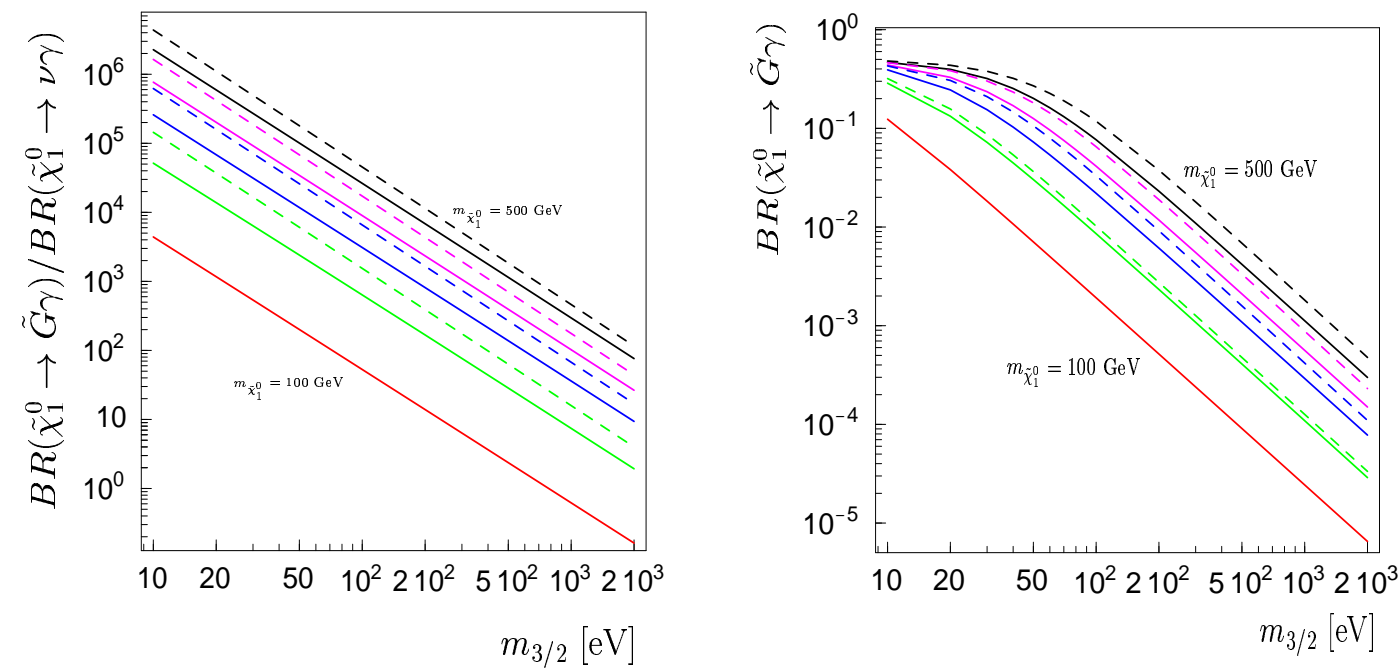

Figure 2: To the left: Ratio $B R\left(\tilde{\chi}_{1}^{0} \rightarrow \tilde{G} \gamma\right) / B R\left(\tilde{\chi}_{1}^{0} \rightarrow v \gamma\right)$ as function of $m_{3 / 2}$ [eV]. Full lines are for $\tan \beta=10$, dashed lines for $\tan \beta=35$. The different lines are for (from bottom to top) $m_{\tilde{\chi}_{1}^{0}}=100-500$ $\mathrm{GeV}$ in steps of $100 \mathrm{GeV}$. To the right: Ratio $B R\left(\tilde{\chi}_{1}^{0} \rightarrow \tilde{G} \gamma\right)$ as function of the gravitino mass, $m_{3 / 2}[\mathrm{eV}]$.

assumptions possible, see fig. (1). Observing ratios of branching ratios outside the range allowed by neutrino data would dictate that the bilinear RPV model is incomplete.

The main result of the current work is shown in fig. (2), to the right, which shows $B R\left(\tilde{\chi}_{1}^{0} \rightarrow\right.$ $\tilde{G} \gamma)$ as function of the gravitino mass for various different values of the lightest neutralino mass. As promised, in the cosmologically interesting range of $m_{3 / 2}$, this decay has a branching ratio of $\sim 10^{-(2-3)}$. The left graph in fig. (2) demonstrates that for the same values of parameters $B R\left(\tilde{\chi}_{1}^{0} \rightarrow v \gamma\right)$, which gives an indistinguishable signature, is expected to be much lower, essentially due to a 1-loop supression.

\section{Discussion}

In case the neutralino is the NLSP and assuming that bilinear R-parity breaking is the origin of neutrino masses and mixings, measuring the branching ratio for the decay $B R\left(\tilde{\chi}_{1}^{0} \rightarrow \tilde{G} \gamma\right)$, i.e. $\tilde{\chi}_{1}^{0} \rightarrow \gamma+E$, implies an indirect measurement for the gravitino mass $m_{3 / 2}$. Such a measurement can be used to constrain light gravitino dark matter.

In GMSB also the lighter scalar tau can be the LSP. Unfortunately in this case there is no clear unequivocal signal for a light gravitino, since the final state $\tilde{\tau}_{1} \rightarrow l_{j} v_{i}$, expected to dominate in $\not R_{p}$ models, is indistinguishable from $\tilde{\tau}_{1} \rightarrow l_{j} \tilde{G}$.

One final comment before closing. The gravitino certainly is not the only dark matter candidate, even if R-parity is violated. For example, if SUSY exists, the (fermionic) superpartner of the axion [14], the axino, could be the DM. An axino LSP, in $\not R_{p}$ models, in many respects behaves similar to a light gravitino: It interacts very weakly, it decays very slowly (although not as slow as the gravitino), etc. However, the decay rate for a $\tilde{\chi}_{1}^{0}$ NLSP via $\tilde{\chi}_{1}^{0} \rightarrow \tilde{a} \gamma$ can be estimated to be [15] 


$$
\Gamma\left(\tilde{\chi}_{1}^{0} \rightarrow \tilde{a} \gamma\right) \simeq \frac{\alpha_{\mathrm{EM}}^{2} C_{a \chi \gamma}^{2}}{128 \pi^{3}} \frac{m_{\tilde{\chi}_{1}^{0}}^{3}}{\left(f_{a} / N\right)^{2}}
$$

Here, $C_{a \chi \gamma}=\left(C_{a Y Y} / \cos \theta_{W}\right) N_{11}$, with $C_{a Y Y}$ a numerical factor depending on the axion model. Unfortunately, for realistic values of $\left(f_{a} / N\right)$, say $\left(f_{a} / N\right) \simeq 10^{11} \mathrm{GeV}$, this turns out to be of order $\Gamma\left(\tilde{\chi}_{1}^{0} \rightarrow \tilde{a} \gamma\right) \sim 10^{-15} \mathrm{eV}$, much too small to be measurable, compared to the $\mathbb{R}_{p}$ channels discussed above. In contrast to the gravitino case, the axino hypothesis can therefore not be constrained by the measurements discussed here.

\section{Acknowledgments}

This work was supported by Spanish grant BFM2002-00345, by the European Commission Human Potential Program RTN network HPRN-CT-2000-00148, by the European Science Foundation network grant N.86 and by a MEC Ramon y Cajal contract. I would like to thank W.Porod and D. Restrepo for a pleasant collaboration.

\section{References}

[1] M. Hirsch, W. Porod and D. Restrepo, JHEP 0503 (2005) 062 [arXiv:hep-ph/0503059].

[2] M. Hirsch, M. A. Diaz, W. Porod, J. C. Romao and J. W. F. Valle, Phys. Rev. D62, 113008 (2000), [hep-ph/0004115]; [Erratum-ibid. D 65 (2002) 119901]; M. A. Diaz, M. Hirsch, W. Porod, J. C. Romao and J. W. F. Valle, D68, 013009 (2003) [hep-ph/0302021]

[3] For a recent review on neutrino oscillation data, see, for example: M. Maltoni, T. Schwetz, M. A. Tortola and J. W. F. Valle, New J. Phys. 6 (2004) 122 [arXiv:hep-ph/0405172].

[4] F. Takayama and M. Yamaguchi, Phys. Lett. B 485 (2000) 388 [arXiv:hep-ph/0005214].

[5] For a review on gauge mediated supersymmetry breaking, see: G. F. Giudice and R. Rattazzi, Phys. Rept. 322 (1999) 419 [arXiv:hep-ph/9801271].

[6] H. Pagels and J. R. Primack, Phys. Rev. Lett. 48 (1982) 223.

[7] S. Eidelman et al. [Particle Data Group], Phys. Lett. B 592 (2004) 1.

[8] E. A. Baltz and H. Murayama, JHEP 0305 (2003) 067 [arXiv:astro-ph/0108172].

[9] M. Fujii and T. Yanagida, Phys. Lett. B 549 (2002) 273 [arXiv:hep-ph/0208191].

[10] W. Porod, M. Hirsch, J. Romao and J. W. F. Valle, Phys. Rev. D 63 (2001) 115004 [arXiv:hep-ph/0011248].

[11] M. Hirsch, W. Porod, J. C. Romao and J. W. F. Valle, Phys. Rev. D 66 (2002) 095006 [arXiv:hep-ph/0207334].

[12] W. Porod, Comput. Phys. Commun. 153 (2003) 275 [arXiv:hep-ph/0301101].

[13] R. Barate et al. [ALEPH Collaboration], Phys. Lett. B 565 (2003) 61 [arXiv:hep-ex/0306033].

[14] For reviews on axions in cosmology, see, for example: J. E. Kim, Phys. Rept. 150, 1 (1987). G. G. Raffelt, Phys. Rept. 198 (1990) 1.

[15] L. Covi, H. B. Kim, J. E. Kim and L. Roszkowski, JHEP 0105 (2001) 033 [arXiv:hep-ph/0101009]. 